\title{
Median Filter Performance Based on Different Window Sizes for Salt and Pepper Noise Removal in Gray and RGB Images
}

\author{
Elmustafa S.Ali Ahmed ${ }^{1}$, Rasha E. A.Elatif ${ }^{2}$ and Zahra T.Alser ${ }^{3}$ \\ Department of Electrical and Electronics Engineering, Red Sea University, Port \\ Sudan, Sudan \\ 1elmustafasayed@gmail.com, ${ }^{2}$ rashamy2002@yahoo.com, \\ 3zahraa1118@hotmail.com
}

\begin{abstract}
Noised image is a major problem of digital image systems when images transferred between most electronic communications devices. Noise occurs in digital images due to transmit the image through the internet or maybe due to error generated by noisy sensors. Other types of errors are related to the communication system itself, since image needed to be transferred from analogue to digital and vice versa, also to be transmitted in most of the communication systems. Impulse noise added to image signal due to process of converting image signal or error from communication channel. The noise added to the original image by changes the intensity of some pixels while other remain unchanged. Salt-and-pepper noise is one of the impulse noises, to remove it a simplest way used by windowing the noisy image with a conventional median filter. Median filters are the most popular nonlinear filters extensively applied to eliminate saltand-pepper noise. This paper evaluates the performance of median filter based on the effective median per window by using different window sizes and cascaded median filters. The performance of the proposed idea has been evaluated in $M A T L A B$ simulations on a gray and RGB images. The experimental results show that median filter has a good performance in gray and RGB images in low noise densities and also in high noise densities when using cascaded median filter and high level of window sizes, but with higher window size a degree of blurring effect will be added to filtered noise.
\end{abstract}

Keywords : Salt and pepper noise, median filter, cascaded filters, window sizes, MSE, PSNR, Gray image, RGB image, linear filtering.

\section{Introduction}

Digital Image Processing becomes a major field of study in the area of computer science and communication engineering that because of the importance of digital image processes in most of the computer and communication systems. All systems which were operating on the traditionally analog imaging are now gradually switching to the digital systems for their ease of use, affordability and flexibility. Due to converting images from analog form to digital form an impulse noise appears during image processing by a camera, scanner, and recording in most types of systems such TV stations or Satellites, and when the image is transmitted over a noisy channel [1]. Image processing is very important and has been extensively used in the area of medicine, film and video production, photography, remote sensing satellites, military target analysis, and manufacturing automation and control [2]. Salt-and-pepper noise is a special case of impulse noise, where a certain percentage of individual pixels are randomly digitized into 
two extreme, intensities, maximum and minimum. The need to remove salt-and-pepper noise is very important before subsequent image processing tasks are carried out because the contamination of image by salt-and-pepper noise is caused in great amount and the occurrence of noise can severely damage the information or data contained in the original image [3]. Noise filtering techniques can either be linear or non-linear. The linear filtering technique applies the algorithm linearly to all the pixels in the image without defining the image as corrupted or uncorrupted pixel. Median filter is most filter used to remove the salt and pepper noise it uses a technique of windowing pixels of noisy image. The pixels in the window are first sorted and the center pixel is changed to the median value of the sorted sequence. This method is the simplest of the median filtering techniques and because of its simplicity [4].

In this work we evaluate the performance of median filter based on different window sizes and study the effects of variation of window size in order to remove the salt and pepper noise from gray image and colored (RGB) image, other experiments also taken to evaluate the performance of cascaded 2 median filters as in case of different widow sizes. The paper is organized as follows; section 2, reviews a type's of impulse noise in digital images. Section 3, discusses the impulse noise removal techniques of using median filter and some of other linear filters and their implementations. Section 4, presents the concept of proposed idea of median filter based on median per different window sizes. Experimental results are shown in section 5 which is divided into several scenarios, Scenario A presents the experiments of median filter evaluation based on different window sizes $3 * 3,5 * 5,7 * 7$ and $9 * 9$ on gray image in order to remove salt and pepper noise. Scenario B reviews the experiments of median filter evaluation on colored (RGB) image and Scenario $\mathrm{C}$ illustrates the performance of 2 cascaded median filters on both Gray and RGB images .Finally the paper conclusion and future work is in section 6.

\section{Impulse Noise Removal Using Filtering}

Filtering is an essential part of any signal processing system, which involves estimation of a signal degraded, in most cases, by additive random noise. Several filtering techniques have been reported over the years, for various applications. In image processing problems, nonlinear filtering techniques are preferred as they can cope with the nonlinearities of the image formation model and also take into account the nonlinear nature of the human visual system [5]. Thus, the filters having good edge and image detail preservation properties are highly desirable for image filtering.

Impulse noise reduction algorithms are broadly classified in to two classes: linear and nonlinear algorithms. Many image de-noising algorithms for correcting the images corrupted by impulse noise are proposed. In a linear technique, the noise reduction method is applied linearly to all the pixels in the corrupted image without checking for the corrupted pixels, whereas in non-linear methods corrupted and no corrupted pixels are determined first then the reduction techniques are applied for correcting the corrupted pixels only [5]. Many types of linear and nonlinear filters presented to noise removal, the most filters used in image noise removal illustrated below.

\section{1-Mean Filter (MMF)}

Mean Filter is a linear filter which odd length fixed sized scanning window is used to get restored image from the corrupted image. With the help of the scanning window corrupted image pixels are scanned in horizontal and vertical directions. In each scan test pixel is replaced by the mean value of the scanning window pixels. 


\section{2- Weighted Mean Filter (WMF)}

Weighted mean filter is a linear filter and another modified basic mean filter. In this filter weights are assigned to scanning window pixels. Mean value of the products of pixel values and their corresponding weights are used as restoration value of the test pixel as shown in equation [5]. Based on different parameters such as distance from the window center pixel, direction and position in ordered statistics, etc. different filters use different ways of weight calculations.

\section{3-Trimmed Mean Filter (TMF)}

Trimmed mean filter is a linear filter known as an improved version of the mean filter where test pixel is replaced by trimmed mean value of the window pixels. To calculate the trimmed mean value of the pixels, trimming value must be known. All available window pixels are arranged in ascending order of their intensity values. From ordered list of pixels first and last pixels are removed and the mean value of the remaining pixels is used as the restoration value for the corrupted pixels. The process of removing the first and the last set of pixels from an ordered list is called trimming.

\section{4-Standard Median Filter (SMF)}

The standard median filter [6] is a nonlinear filter called as median smoother that attempts to remove impulse noise by changing the luminance value of the center pixel of the filtering window with the median of the luminance values of the pixels contained within the window. Although the median filter is simple and provides a reasonable noise removal performance, it removes thin lines and blurs image details even at low noise densities.

\section{5-Weighted Median Filter (WMF)}

Weighted median filter is one of the branch of median filter (WMF) .The operations involved in WMF are similar to SMF, except that WMF has weight associated with each of its filter element these weights correspond to the number of sample duplications for the calculation of median value.

\section{6-Adaptive Median Filter (AMF)}

The Adaptive Median Filter is designed to eliminate the problems faced with the standard median filter. The basic difference between the two filters is that, in the Adaptive Median Filter, the size of the window surrounding each pixel is variable. This variation depends on the median of the pixels in the present window. If the median value is an impulse, then the size of the window is expanded [7].

\section{Median Filter Algorithm}

The median filter is a nonlinear digital filtering technique, often used to remove salt and pepper noise. Median filtering is very widely used in digital image processing because, under certain conditions, it preserves edges of the images while removing noise and it's a non-linear local filter whose output value is the middle element of a sorted array of pixel values from the filter window. The median filter filters each pixel in the image in turn and its nearby neighbors are used to decide whether or not it is representative of its surroundings [8]. Median filter replaces the pixel value with the median of those values. That is, the values from the surrounding neighborhood are first sorted into numerical order, and then the value of the pixel in question is replaced with the middle (median) pixel value. The neighborhood is referred to as the window. The window can have various shapes centered on the target pixel, typical a square shape chosen for windows defined for 
$2 \mathrm{D}$ images. The median value of the surrounding pixels is most likely to be the value of one of the pixels in the neighborhood within the window. Thus the median filter is least likely to create new unrealistic pixel values. For this reason, the median filtering technique is much better than the mean filtering technique in terms of preserving sharp edges [9].

At the pixels on the far horizontal or vertical boundary there is no entry in all directions so zero values are placed to handle the missing window entries at the boundaries of the image. The main drawback of a Standard Median Filter (SMF) is that it is more effective only for low noise densities. At high level noise densities, SMFs often exhibit blurring for large window sizes and insufficient noise suppression for small window sizes. However, most of the median filters operate uniformly across the image and thus tend to modify both noise and noise-free pixels. Consequently, the removal of impulse often leads to images with blurred and distorted features. Ideally, the filtering should be applied only to corrupted pixels while leaving uncorrupted pixels intact. Applying median filter unconditionally across the entire image would inevitably alter the intensities and remove the signal details of uncorrupted pixels. Therefore, a noise detection process to discriminate between uncorrupted pixels and the corrupted pixels prior to applying nonlinear filtering is highly desirable [10].

An important shortcoming of the median filter is that its output is always constrained, by definition, to be the median value in the window. In a $\mathrm{k} \times \mathrm{k}$ window, if the number of polluted pixels is very large, then the median computed will be an impulse and the noise will not be removed. On the other hand the center value replaced is not tested to find out if it is an impulse or not. Hence if it is not an impulse but a fine pixel of the image then it is removed unnecessarily. The median filter performs poorly when the intensity of the noise is high [11].

\section{Simulation And Experimental Results}

Our proposed idea is to analysis the performance of median filter ability to remove the salt and pepper noise in digital images. Median filter performance obtained after simulate the filter to remove noised from gray images and RGB images. Salt and pepper noise added to the images using different noise from density 20 to 70 percentages based on the effect of variation of window size in median filter.

The simulation was divided into four scenarios; scenario A represents the performance of the median filer in gray images, scenario B for RGB images, scenario C represents a performance of using two cascaded median filter in gray and RGB noised images and scenario D for comparison between the effects of the median filter in Gray and RGB images. The performance metrics used are mean square error (MSE) and power signal to noise ratio (PSNR) mathematically represented prospectively as follows;

$$
\begin{gathered}
\mathrm{MSE}=\frac{1}{M N} \sum_{i-1}^{M} \sum_{j-1}^{N}(R i j-X i j)^{2} \\
\mathrm{PSNR}=10 \log 10\left(\frac{255^{2}}{M S E}\right)
\end{gathered}
$$

Where r: represents original image, $\mathrm{x}$ : restored image and $\mathrm{M}^{*} \mathrm{~N}$ : size of the image.

\section{1- Scenario A: Median Filter for Noised Gray Image.}

In scenario a simulation of median filter performance is obtained by using on filter to remove salt and pepper noise form Gray images noised by percentage of 20,30, 40, 50, 60 , and $70 \%$ based on window size used to filtering image in median filter. The results PSNR obtained from simulation is shown in table 1 below. 


\section{Table 1. PSNR (dB) Values for Loin Image (Gray) Using Median Filter}

\begin{tabular}{|c|c|c|c|c|}
\hline $\begin{array}{c}\text { Noise } \\
\text { Density }\end{array}$ & $\begin{array}{c}\text { Window } \\
3 * 3\end{array}$ & $\begin{array}{c}\text { Window } \\
5 * 5\end{array}$ & $\begin{array}{c}\text { Window } \\
7 * 7\end{array}$ & $\begin{array}{c}\text { Window } \\
9 * 9\end{array}$ \\
\hline $20 \%$ & 15.2213 & 14.9381 & 14.7997 & 14.7296 \\
\hline $30 \%$ & 13.5846 & 13.2755 & 13.1733 & 13.1855 \\
\hline $40 \%$ & 12.5306 & 12.2885 & 12.1409 & 11.9934 \\
\hline $50 \%$ & 11.8135 & 11.4818 & 11.3985 & 11.2967 \\
\hline $60 \%$ & 11.1152 & 10.9387 & 10.8815 & 10.7880 \\
\hline $70 \%$ & 10.7174 & 10.4939 & 10.3916 & 10.2939 \\
\hline
\end{tabular}

The gray image of loin is chosen to be noised by salt and paper noise and filtered using median filter, the following images shows the effect of the median filter over gray image based on noise density.

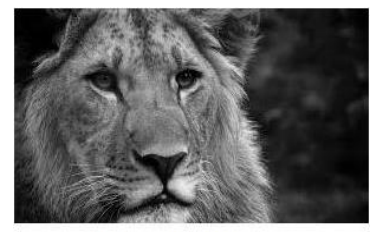

Fig A.1 Original Image

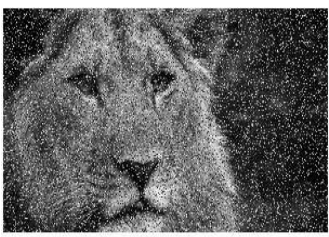

Fig A.2 Noised Image 20\%

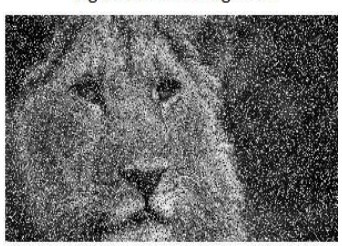

Fig A.3 Noised Image $30 \%$

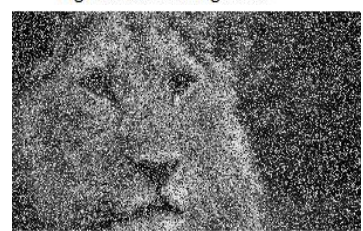

Fig A.4 Noised Image 40\%

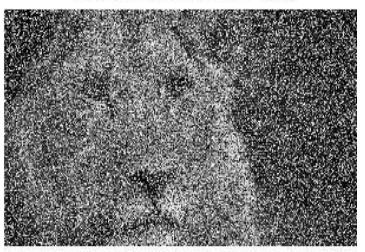

Fig A.5 Noised Image 50\%

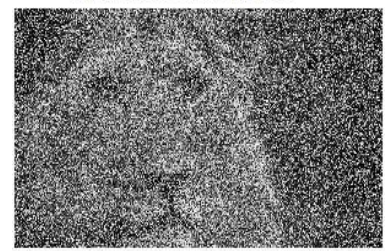

Fig A.6 Noised Image 60\%

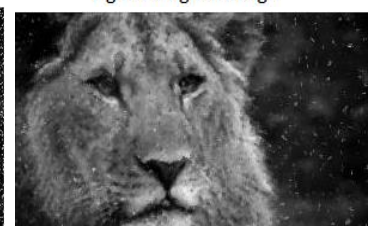

Filtered Image by Window $3 * 3$

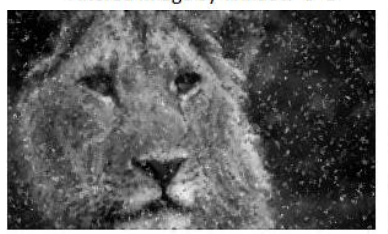

Filtered Image by Window $3 * 3$

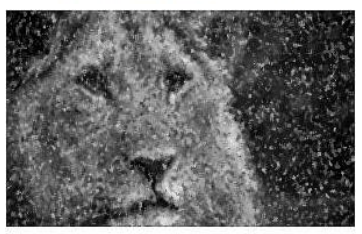

Filtered Image by Window $3^{*} 3$

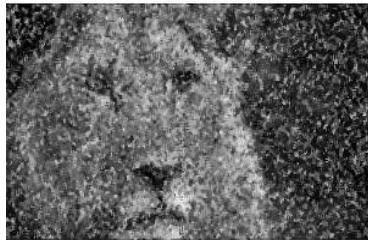

Filtered Image by Window $3 * 3$

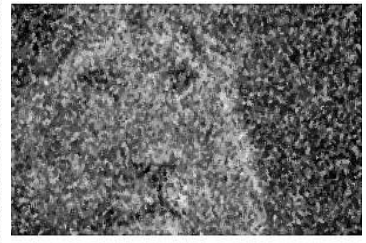

Filtered Image by Window $3 * 3$

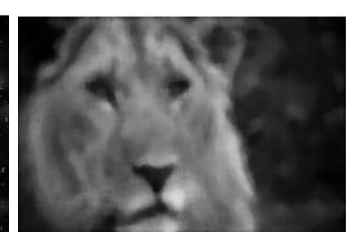

Filtered Image by Window $9 * 9$

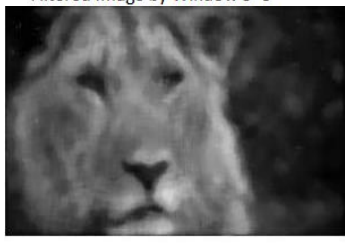

Filtered Image by Window $9 * 9$

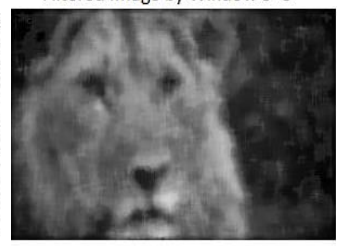

Filtered Image by Window $9 * 9$

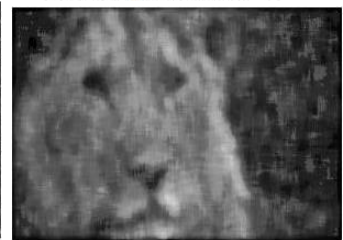

Filtered Image by Window $9 * 9$

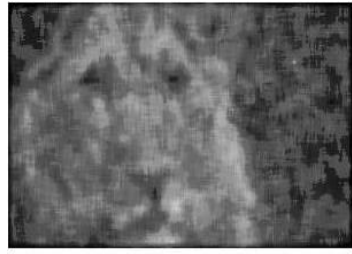

Filtered Image by Windows $9 * 9$ 


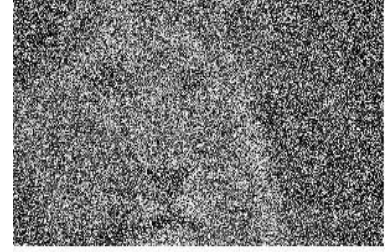

Fig A.7 Noised Image 70\%

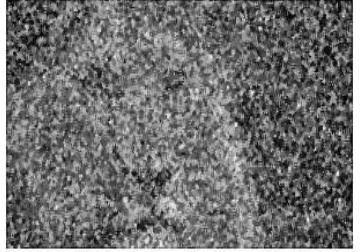

Filtered Image by Window $3 * 3$

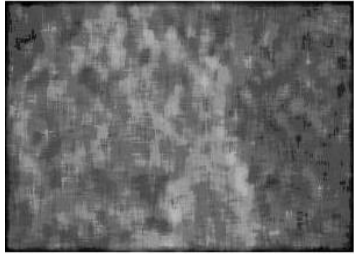

Filtered Image by Windows $9 * 9$

The performance of the median filter to remove salt and pepper noise is calculated by the MSE and PSNR as in the following figures.

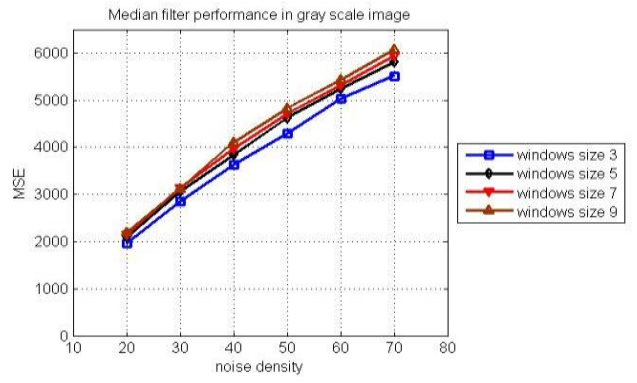

Figure A.8. MSE Performance on Gray Image

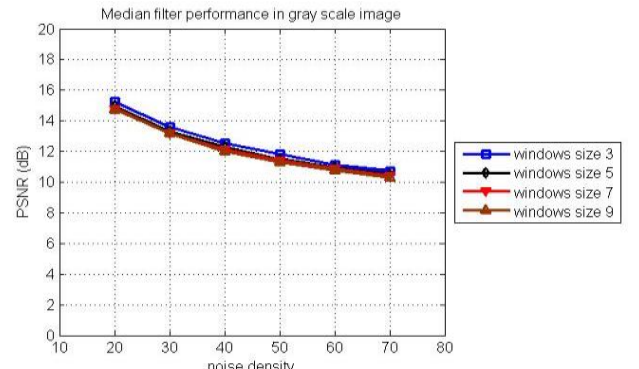

Figure A.9. PSNR Performance on Gray Image

For Gray noised image of Loin as shown in figure A.8 the mean square error values of median filter window sizes $3 * 3,5 * 5,7 * 7$ and $9 * 9$ are closed together in low noise density $20 \%$ but when the noise density is increased to $70 \%$ the MSE of widow size $3 * 3$ is much better than other window sizes, that because when using any window sizes with low noise density the median filter gives a stable performance but due to noise density increasing the median values of window sizes more than $3 * 3$ and $5 * 5$ until to $9 * 9$ will increased and may degrade the hit ratio of median value from all window values specially with high level sizes for example $9 * 9$ which leads to generate a small edge noise appears in the image make MSE becomes higher in high filter window sizes.

The power signal to noise ratio PSNR in figure A.9 show that median filter with window size $3 * 3$ gives higher PSNR than all other window sizes. Overall PSNR is better when using median filter window sizes in low noise densities and PSNR decreases when noise densities increased. For higher window sizes the performance of median filter becomes lower when compared with lower window sizes that because, in low window size the number of elements in windows are dedicated and the median values are closed together which gives a median value of removed noised exactly equal to original value. But when median window increased the elements of median will also increase gives a degree of noise removal but will added a value of new blurring noise in the filtered image. The performance analysis found that the median filer gives best performance for all noised densities in window size $3 * 3$, but with noise density increase and window size increase the median performance remains better only in noise density $40 \%$ for all window sizes.

\section{2- Scenario B: Median Filter for RGB Image}

In scenario B simulation of median filter performance is obtained for RGB images noised by percentage of 20 to $70 \%$ based on window size. The results PSNR obtained from simulation is shown in table 2 below. 
Table 2. PSNR (dB) Values for Book Image (RGB) Using Median Filter

\begin{tabular}{|c|c|c|c|c|}
\hline $\begin{array}{c}\text { Noise } \\
\text { Density }\end{array}$ & $\begin{array}{c}\text { Window } \\
3 * 3\end{array}$ & $\begin{array}{c}\text { Window } \\
5 * 5\end{array}$ & $\begin{array}{c}\text { Window } \\
7 * 7\end{array}$ & $\begin{array}{c}\text { Window } \\
9 * 9\end{array}$ \\
\hline $20 \%$ & 12.0874 & 11.9284 & 11.8680 & 11.8555 \\
\hline $30 \%$ & 10.3414 & 10.2053 & 10.1353 & 10.0477 \\
\hline $40 \%$ & 9.0728 & 8.9712 & 8.8904 & 8.9092 \\
\hline $50 \%$ & 8.0912 & 8.0325 & 7.9878 & 7.9303 \\
\hline $60 \%$ & 7.2347 & 7.2017 & 7.2031 & 7.1401 \\
\hline $70 \%$ & 6.4996 & 6.4029 & 6.4987 & 6.4873 \\
\hline
\end{tabular}

The RGB image of colored books is used, noised by salt and pepper with a several noise density ratio then noised image filtered by median filter, the effect of median filter for noise removal in RGB images shown in the following images.

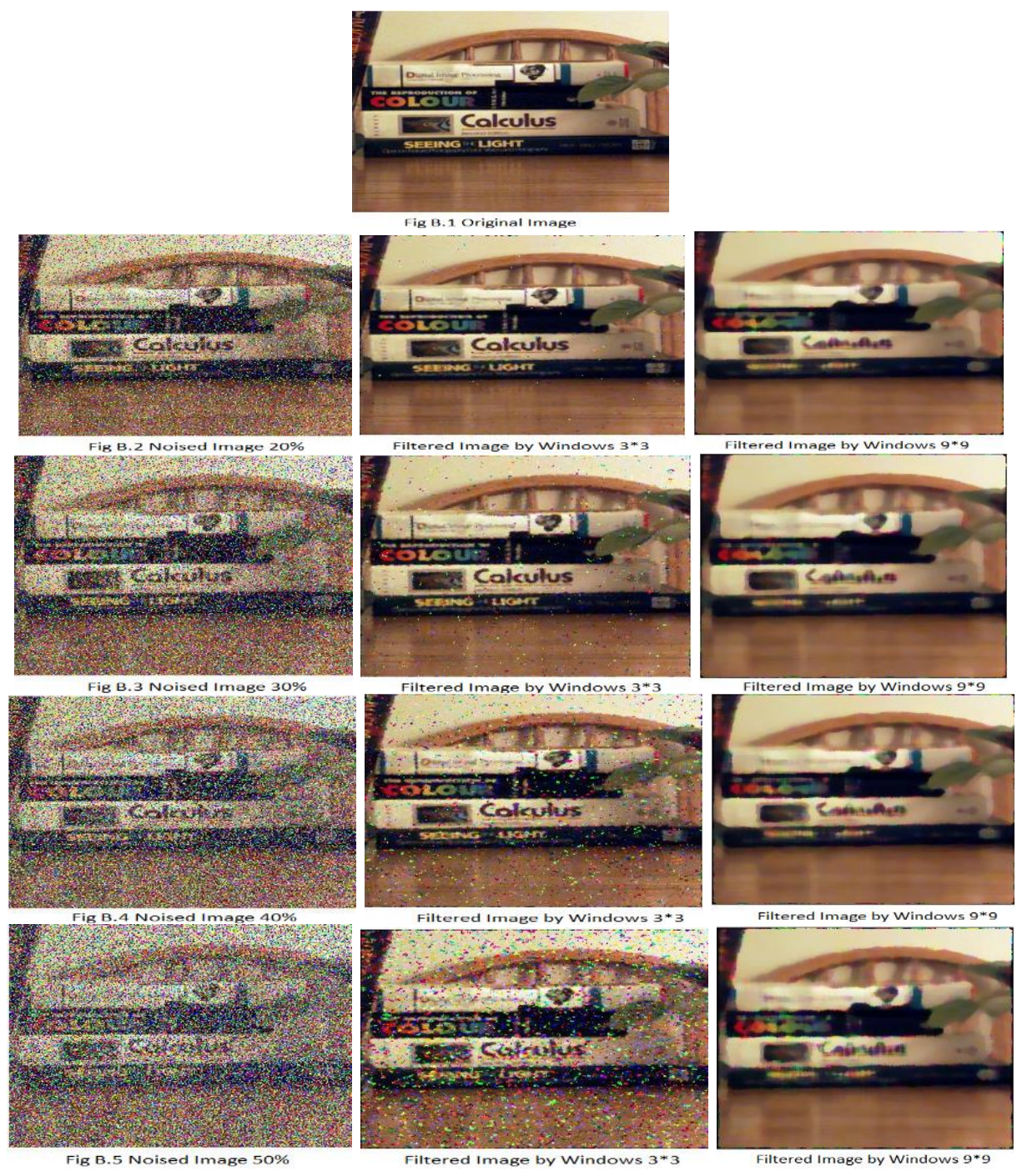




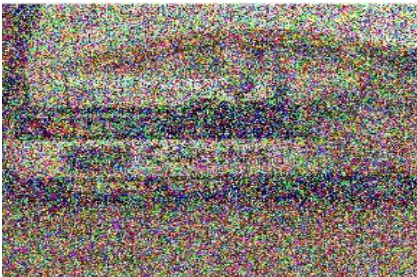

Fig B.6 Noised Image $60 \%$

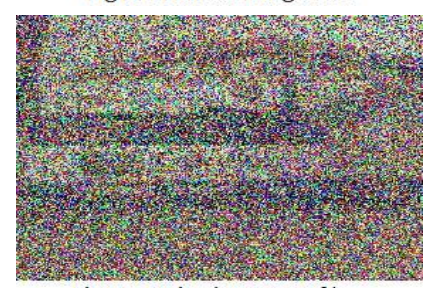

Fig B.7 Noised Image 70\%
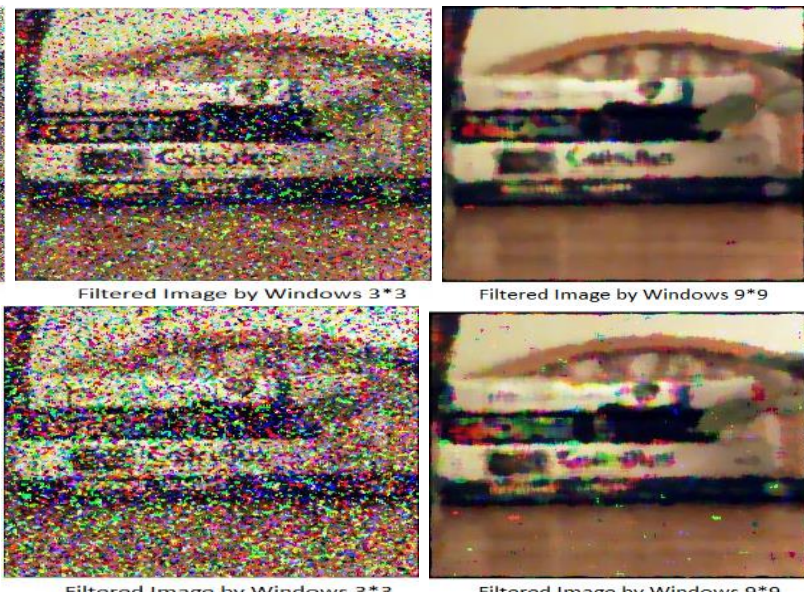

Filtered Image by Windows $3 * 3$
Filtered Image by Windows $9 * 9$

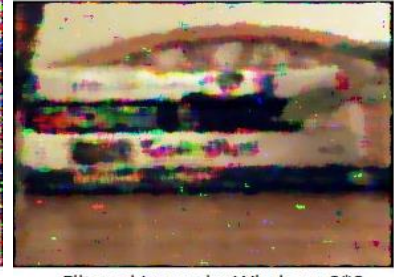

The performance of the median filter to remove salt and pepper noise from RGB image is calculated by the MSE and PSNR and shown as in the following figures.

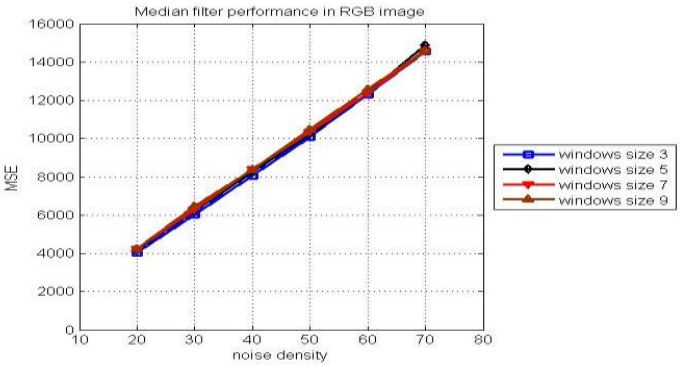

\section{Figure B.8. MSE Performance on RGB Image}

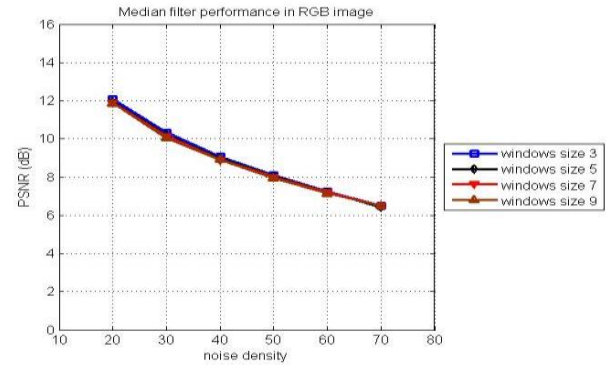

Figure B.9. PSNR Performance on RGB Image

For RGB image the performance of median filter with different window sizes gives close values of MSE in low and high noise densities as shown in figure B.8, that because colored images affected by salt and pepper noise in three set of values red, green and blue, the median values of the three colors gives a median element values close to original noised images due to close values of color degree. The PSNR of all window sizes are also closed together as in figure B.9, the values of median filter window sizes are close together because of the degree of colors in image which leads to stable performance of median filter in all window sizes. For overall median performance could be increased if the window size is increased, but as the window size increases, the image tends to show blurring effect. But as the noise density increases further, the median filter lead to lower performance. On the other hand, median filtering shows the best performance for all noise densities. Upon analysis, it is found that it can filter the salt and pepper noise for densities up to $60 \%$ without any blurring effect on the filtered image. But for larger noise densities, the blurring of the filtered images tends to increase.

\section{3- Scenario C: Cascaded Median Filter for Gray and RGB Images}

This scenario evaluates the performance of cascaded median filter for salt and pepper noise removal, the two median filters connected in series way and then used to removes the salt and pepper noise from Lion Gray image and RGB book image. The evaluation of two cascaded median filter calculated by MSE and PSNR, the output performance is gives in the following figures. 


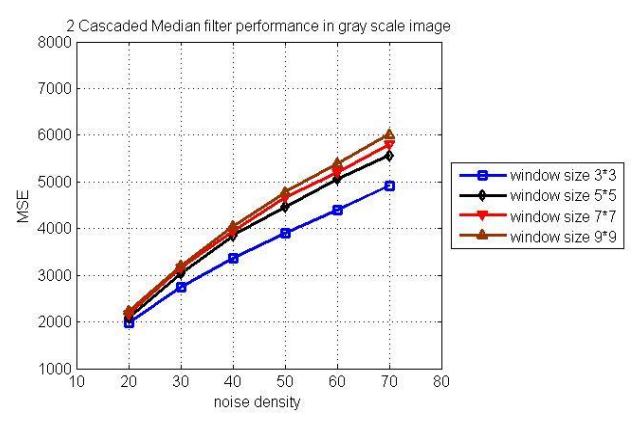

Figure C.1. MSE Performance on Gray Image

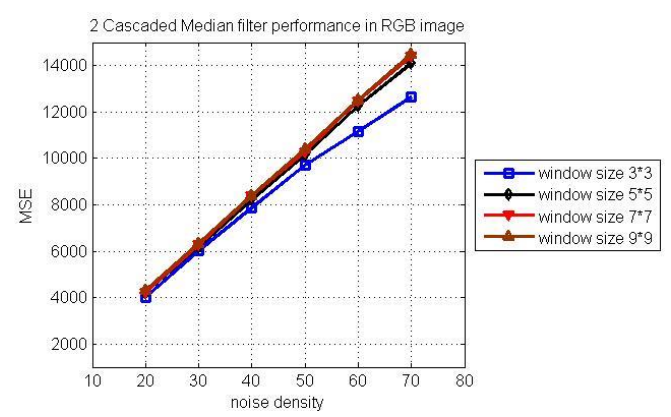

\section{Figure C.3. MSE Performance on RGB Image}

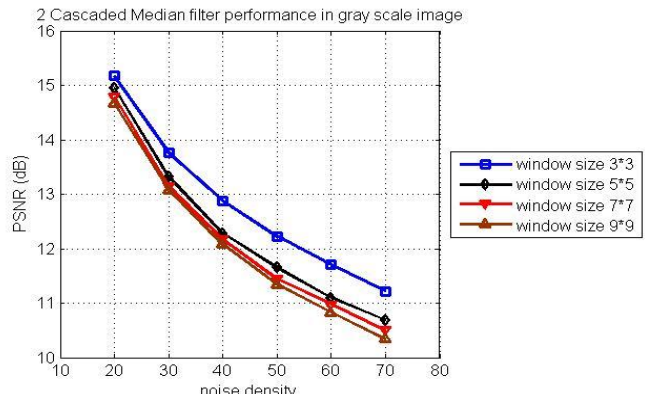

Figure C.2. PSNR Performance on Gray Image

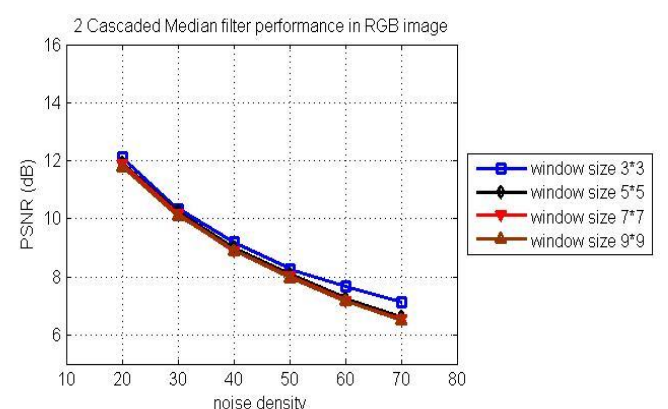

Figure C.4. PSNR Performance on RGB Image

The performance of using two cascaded median filter is much better that one median filter when compare the obtained figures in scenarios A and B with figures C.1, C.2, C.3 and C.4. The MSE of two cascaded median filer in figures C.1 and C.3 for gray and RGB images is same in low noise density $20 \%$, but with high noise density $70 \%$ the median filter with window size $3 * 3$ gives better performance than other window sizes specially in gray images. In figures C.2 and C.4 the PSNR values of all window sizes for two cascaded median filter are closed together in low noise density of $20 \%$ in RGB image but window size of $3^{*} 3$ gives slightly higher performance than other sizes gray image. As noise density increased to $70 \%$ median filter of window size $3 * 3$ in gray image is better than in RGB image.

\section{Conclusion And Future Work}

In this paper a median filter performance has been evaluated in order to remove salt and pepper noise in gray and RGB images, The main contribution of this paper is to evaluate the performance of median filter based on window sizes and its ability to remove different noise densities in gray and colored images. Experimental results show that the window sizes of median filter gives a degree of effect on noise removal in images, low level window size gives better performance in low noise densities for example $3 * 3$ but when noise density increasing the low window sizes ability to remove noise will be degraded, and when using a high level of window sizes such $9 * 9$ the PSNR of filtered images is better even in high noise density but will added a degree of blurring effect on the images. Two cascaded median filter gives higher performance than using one median filter but if the cascaded number of filters increased the performance becomes constant .for future work we propose a new idea to evaluate the adaptive noise filter performance in gray and RGB images compared with standard median filter. 


\section{References}

[1] S. Singh and N. R. Prakash, "Modified Adaptive Median Filter for Salt \& Pepper Noise", International Journal of Advanced Research in Computer and Communication Engineering, vol. 3, no. 1, (2014) January.

[2] W. Wang, M. N. S. Swamy and M. O. Ahmad, "RNS Application for Digital Image Processing," 4th IEEE international workshop on System-on-chip for Real-time Application, (2004), pp. 77-80.

[3] B. Deshpande, H. K. Verma and P. Deshpande, "Fuzzy Based Median Filtering for Removal of Salt-andPepper Noise", International Journal of Soft Computing and Engineering (IJSCE), ISSN: 2231-2307, vol. 2, no. 3, (2012) July.

[4] S. Shrestha, "IMAGE DENOISING USING NEW ADAPTIVE BASED MEDIAN FILTER", Signal \& Image Processing: An International Journal (SIPIJ), vol. 5, no. 4, (2014) August.

[5] T. Gebreyohannes and D.-Y. Kim, "A DAPTIVE NOISE REDUCTION SCHEME FOR SALT AND PEPPER”, Signal \& Image Processing: An International Journal (SIPIJ), vol. 2, no. 4, (2011) December.

[6] S. Athi Narayanan, G. Arumugam and K. Bijlani, "Trimmed Median Filters for Salt and Pepper Noise Removal", International Journal of Emerging Trends \& Technology in Computer Science (IJETTCS), vol. 2, no. 1, (2013) January -February.

[7] M. S. Nair, K. Revath and R. Tatavarti, "Removal of Salt-and Pepper Noise in Images: A New DecisionBased Algorithm", Proceedings of the International Multi Conference of Engineers and Computer Scientists, vol 1, (2008), Hong Kong.

[8] A. Srinagesh, B R L K Dheeraj and G. P. Saradhi Varma, "A New Method for Removal of Salt and Pepper Noise through Advanced Decision Based Unsymmetric Median Filter", (IJCSIT) International Journal of Computer Science and Information Technologies, vol. 5, no. 6, (2014).

[9] V. Ambule, M. Ghute, K. Kamble and S. Katre," Adaptive Median Filter for Image Enhancement", International Journal of Engineering Science and Innovative Technology (IJESIT) vol. 2, no. 1, (2013) January.

[10] V. Katkovnik, K. Egiazarian and J. Astola, "Adaptive Window Size Image De-noising Based on Intersection of Confidence Intervals (ICI) Rule", Journal of Mathematical Imaging and Vision vol. 16, 223 \pm 235 , (2002).

[11] B. Deka and S. Choudhury, "A Multiscale Detection based Adaptive Median Filter for the Removal of Salt and Pepper Noise from Highly Corrupted Images", International Journal of Signal Processing, Image Processing and Pattern Recognition, vol. 6, no. 2, (2013) April.

\section{Authors}

Elmustafa Sayed Ali Ahmed, he received his M.Sc. degree in electronic engineering, Telecommunication from Sudan University of science and technology in 2012, and B.Sc. (Honor) degree in electrical engineering, Telecommunication from Red Sea University in 2008. He was a wireless networks (Tetra system, Wi-Fi and Wi-Max) engineer in Sudan Sea Port Corporation for four years and a head department of electrical and electronics engineering, faculty of engineering in Red Sea University for one year. He published papers, chapters, and books in wireless communications, computer and networking in peer-reviewed academic international journals. His areas of research interest include MANETs, wireless networks, VANETs, image processing and computer networks.

Rasha Eltayeb Abd Elatif , she received her B.Sc. degree in aeronautical engineering, avionics from Sudan university of science and technology in 2006. She was a teacher assistant for one year in Sudan university of science and technology 2007-2008 then she worked as technical engineer in Sudan university of science and technology engineering college aeronautical department from 2008 to present. She mandated to Red Sea university department of electrical engineering since 2012. Her research interests includes on DSP, Mobile Networks and Routing Protocols.

Zahraa Tag Elsir, she received her B.Sc. degree in telecommunication engineering from university of Khartoum in 2012. She is a teacher assistant in electrical and electronics engineering department, in Red Sea University until now. Her research interests are on DSP, Mobile Communication and Satellites Networks. 